\title{
An extensible mathematical model of glucose metabolism. Part I: the basic glucose-insulin-glucagon model, basal conditions and basic dynamics
}

\author{
Caleb L. Adams ${ }^{a}$ (D) and D. Glenn Lasseigne ${ }^{b}$ \\ ${ }^{a}$ Department of Mathematics and Statistics, Radford University, Radford, VA, USA; ${ }^{b}$ Department of \\ Mathematics and Statistics, Old Dominion University, Norfolk, VA, USA
}

\begin{abstract}
A basic model highlighting the counter-regulatory roles of insulin and glucagon is proposed to start a series of models designed to explore continuous rein control and major aspects of glucose metabolism. The three-by-three dynamical system uses black boxes to model unit processes such as the dependencies of insulin secretion rate and the glucagon secretion rate on blood glucose concentration. The dependency of basal conditions on insulin resistance and any defects in insulin or glucagon secretion are shown. Since over-production of hepatic glucose exists early in the history of diabetes, it is important that mathematical models should account for this effect by inclusion of the dynamical equation governing glucagon concentration as this illustrative model does. All solutions are consistent with gross features of the metabolic process. The model is examined for explicit and implicit assumptions affecting its validity which determines that the first extension to the model should account for glucose storage and the release of stored glucose.
\end{abstract}

\section{ARTICLE HISTORY}

Received 24 February 2017

Accepted 7 January 2018

\section{KEYWORDS}

Glucose metabolism; basal conditions; continuous rein control

\section{Introduction}

The healthy state of the human body depends on tightly regulated glucose metabolism. Two important counter-regulatory hormones of glucose metabolism include insulin and glucagon. In a healthy individual, the glucose regulatory system manages the body's blood glucose concentration (typically between 70 and $120 \mathrm{mg} / \mathrm{dl}$ ) despite input from eating and additional use of internal glucose stores. Defects in this regulatory system, if left untreated or poorly treated, lead to a life-threatening disease state (Type I diabetes) or may lead to long-term complications, ill-health and possible early death (impaired fasting glucose tolerance and insulin resistance leading to Type II diabetes).

In order to better understand a complex biological system such as glucose metabolism, many researchers have relied upon mathematical modelling. Beginning with a model using ordinary differential equations by Bolie (1961), mathematicians and scientists have

CONTACTCaleb L.Adams cadams5@radford.edu 
been developing models for over the past five decades. Other early attempts to describe glucose regulation appeared in several models during the 1960s and 1970s (Ackerman, Gatewood, Rosevear, \& Molnar, 1965; Della et al., 1970; Serge et al., 1973; Srinivasan et al., 1970). In 1979, Bergman, Ider, Bowden, and Cobelli published one of the most influential models of glucose regulation - commonly known as the 'minimal model' - highlighting glucose disappearance following the intravenous glucose tolerance test (IVGTT). The minimal model incorporates two ordinary differential equations to govern the plasma glucose concentration, $G$, and the concentration in a compartment remote from the plasma, $X$, and are given as

$$
\begin{aligned}
& \frac{d G}{d t}=\left(p_{1}-X\right) G+p_{4} \\
& \frac{d X}{d t}=p_{2} X+p_{3} I(t) .
\end{aligned}
$$

$I(t)$ represents the measured plasma insulin and the $p_{i}$ are constants determined by matching the model's solution to the observed glucose profile. Using the measured basal glucose value as an essential parameter in the system, the model provided a good fit to the short-term data from the IVGTT.

The following year saw improvements in incorporating insulin's role in models of glucose metabolism (Toffolo et al., 1980) and further testing and validation of the minimal model by Bergman, Phillips, and Cobelli (1981) followed shortly thereafter. Several reviews have been published featuring advancements made from the minimal model (Ajmera, Swat, Laibe, Le Novère, \& Chelliah, 2013; Bergman, 2002; Bergman, Finegood, \& Ader, 1985; Boutayeb \& Chetouani, 2006). These reviews provide a comprehensive list of articles for interested readers which present adaptations and enhancements to the minimal model. The usefulness of the minimal model approach in physiological research is in providing mathematical models with the fewest equations and parameters necessary to provide solution profiles which fit the observed short-term profiles of glucose and insulin in a variety of experiments. The models are often tuned to each different experiment. By definition, these models are too simplistic to capture long-term phenomena.

In contrast to the minimal model approach, comprehensive mathematical models have been developed to explain more complex long-term behaviour. For example, two distinct period ranges have been observed for the oscillations in insulin secretion: rapid, 10-15 min, and ultradian, 100-150 min. One of the earliest models addressing the biphasic nature of insulin secretion was developed by Sturis et al. (1991). Their model incorporated a system of non-linear ordinary differential equations to simulate the ultradian oscillations that occur in the glucose-insulin feedback system:

$$
\begin{aligned}
\frac{\mathrm{d} x}{\mathrm{~d} t} & =f_{1}(z)-E\left(\frac{x}{V_{1}}-\frac{y}{V_{2}}\right)-\frac{x}{t_{1}}, \\
\frac{\mathrm{d} y}{\mathrm{~d} t} & =E\left(\frac{x}{V_{1}}-\frac{y}{V_{2}}\right)-\frac{y}{t_{2}}, \\
\frac{\mathrm{d} z}{\mathrm{~d} t} & =f_{5}\left(h_{3}\right)+I-f_{2}(z)-f_{3}(z) f_{4}(y), \\
\frac{\mathrm{d} h_{1}}{\mathrm{~d} t} & =\frac{3\left(x-h_{1}\right)}{t_{3}}
\end{aligned}
$$




$$
\begin{aligned}
\frac{\mathrm{d} h_{2}}{\mathrm{~d} t} & =\frac{3\left(h_{1}-h_{2}\right)}{t_{3}}, \\
\frac{\mathrm{d} h_{3}}{\mathrm{~d} t} & =\frac{3\left(h_{2}-h_{3}\right)}{t_{3}}
\end{aligned}
$$

where $x$ is the amount plasma insulin, $y$ is the amount of remote insulin, $z$ is the amount of glucose, $E$ is a constant rate of insulin exchange between compartments, $V_{1}, V_{2}$, and $V_{3}$ are volumes, $f_{1}(z)$ represents insulin secretion, $f_{2}(z)$ represents insulin-independent glucose utilization, while $f_{3}(z)$ and $f_{4}(y)$ are insulin-dependent utilizations, $f_{5}\left(h_{3}\right)$ represents glucose production, $h_{1}, h_{2}$, and $h_{3}$ are variables representing delay processes between plasma insulin and glucose production, $I$ is the exogenous glucose delivery rate, $t_{1}$ and $t_{2}$ are time constants related to insulin degradation, and $t_{3}$ is the delay time between plasma insulin and glucose production. It is not clearly understood from where the oscillations are generated. The authors identified a difference between simulations and experimental data. They suggest that glucose plays an important role in the generation of insulin oscillations and propose that abnormal ultradian oscillations may be due to glucose intolerance.

Despite defects in insulin secretion, Leahy (1990) reports that normoglycemia may be maintained in individuals with type 2 diabetes, at the cost of elevated insulin levels. Diet, exercise, and drug intervention are the major treatments used to combat these defects. Some individuals with impaired fasting glucose tolerance are able to restore an almost normal blood glucose control through diet and exercise alone (Clark, 1997; GoldhaberFiebert et al., 2003; Horton, 1988). Physical exercise has been identified as both a catalyst for increasing glucose uptake by the muscles and impacting insulin sensitivity (Borghouts \& Keizer, 2000; Goodyear \& Kahn, 1998). Derouich and Boutayeb (2002) proposed an adaptation to Bergman's minimal model investigating the effects of physical activity on the dynamics of glucose and insulin levels but admit that the data used to derive parameters was based upon studies from healthy subjects. Roy and Parker (2007) explored effects of both short- and long-term exercise on plasma glucose and insulin levels during the postexercise recovery period. Numerical analysis of a model using two differential equations with a delay element and inclusive external periodic functions defining diet and physical exercise demonstrated stable periodic solutions exist for glycaemia and insulin dynamics in both normal and diabetic cases (Švitra et al., 2010). Typically, Type II diabetics require pharmacological intervention.

The importance of understanding the mechanisms of glucose metabolism is obvious for individuals in ill health; however, the mechanisms of glucose metabolism underlie a current debate that is of importance for non-diabetics: Is a severely restricted low-carbohydrate diet appropriate for humans? An evaluation by Sylvetsky et al. (2016) concluded that higher intake of carbohydrate from vegetables and fibre and lower intake of sweets and soda was associated with lower diabetes risk factors. Proponents of such diets note the sharp rise in insulin concentrations following a high-carbohydrate meal and insulin's promotion of energy storage for the eventual weight gain of an individual. To monitor such intakes, dieters following a low-carbohydrate lifestyle utilize the glycemic index of foods in order to choose a diet that prevents large after-meal increases in the blood glucose concentration (Brand-Miller, 2003). The glycemic index compares the change in blood glucose concentration in an individual after the consumption of a standard quantity of 
carbohydrate to the change in blood glucose concentration in an individual after the consumption of a same quantity of pure glucose.

Because foods with low glycemic index do not quickly raise the blood sugar to high levels, the insulin release in a healthy individual is less than the corresponding insulin release for the same quantity of food with a high glycemic index. However, Sheard et al. (2004) conclude that restricting total carbohydrate consumption to less than $130 \mathrm{~g} /$ day is not recommended as the brain and central nervous system require glucose as an energy source. In addition, they conclude that if an individual is considering only the total amount of carbohydrates consumed, then the glycemic index is a useful tool for blood glucose regulation; but, the individual needs to also consider the type of carbohydrate that is consumed.

Equally important to how the body responds to the intake of food is the way the body maintains glucose levels during the fasting state through release of stores in the liver to compensate for continued metabolism by the central nervous system and muscle tissue. Tirosh et al. (2005) report in a large study that young men with fasting plasma glucose levels in the high-normal range (95-99 $\mathrm{dl} / \mathrm{mg})$ are at least three times more likely to develop Type II diabetes than those with fasting plasma glucose levels in the low-normal range (less than $81 \mathrm{dl} / \mathrm{mg}$ ). The results suggest that over-production of hepatic glucose exists early in the history of diabetes and is exaggerated by obesity. Upon examining this limited introduction to glucose metabolism, three questions are identifiable with answers illustratable through use of mathematical simulation: (a) How does total insulin release correspond to the glycemic index? (b) What relevance does the glycemic index have for blood sugar control in individuals with impaired fasting glucose tolerance or Type II diabetes? and (c) What does the basal blood glucose concentration indicate about the individual's chance to develop Type II diabetes?

The ultimate goal of the investigation is a mathematical model capable of qualitatively describing the long-term metabolism within the body. Proposed in this article is an extensible mathematical model designed to mimic the basic glucose-insulin-glucagon counterregulatory system. At a minimum, the model should eventually include the following effects:

(a) Base Effects

- increased insulin secretion with increasing plasma-glucose concentration,

- increased glucagon secretion with decreasing plasma-glucose concentration,

- maintenance of near-constant concentrations during the postabsorptive period,

(b) Glycogen and Substrate Effects

- dependence of metabolism on stores of glycogen in the liver,

- dependence of metabolism on stores of gluconeogenetic substrates,

- dependence of metabolism on stores of glycogen in the muscle and other tissue,

- production of gluconeogenetic substrates by metabolism of glucose and glycogen in the muscle and other tissue (especially during exercise),

(c) Adipose Tissue Effects

- conversion of excess glucose to fat in adipose tissue,

- conversion of fat to glucose,

and 
(d) Secretion and Compartment Movements

- effects of changes in $\beta$-cell mass over time,

- details of phasic secretion of insulin accounting for a pool of rapidly releasable insulin,

- movement of insulin through the various compartments of the body,

- movement of glucose through the various compartments of the body.

This report presents a model accounting for the base effects enumerated above, which represents the simplest possible starting point for a model describing the long-term dynamics of glucose regulation. Future reports will present progressive enhancements of the model. By proceeding in this manner, the effect of each model component is understood.

The next section will highlight a related model which served as motivation for the proposed model. Section 3 details the construction of the proposed model and necessary fundamentals. Section 4 focuses on the fundamental parameters for the model presented. Sections 5 and 6 will, respectively, illustrate how basal conditions and other set points associated with the model are derived from physiological parameters, and briefly discuss future steps to be taken by the authors.

\section{Model motivation}

The mathematical modelling of glucose metabolism has existed for nearly five decades. The development of 'minimal models,' including those relating insulin and glucose (Bergman, Ider, Bowden, \& Cobelli, 1979; Matthews et al., 1985), are useful for describing basic interactions within complex biological systems. The proposed basic glucose-insulin-glucagon model updates the illustrative model of Saunders et al. (1998) to more accurately model the regulatory process. They introduced the principal of rein control in which regulation of blood glucose is directly effected by both insulin and glucagon. The model they propose tracks changes in glucose $(G)$, glucagon $(A)$, and insulin $(B)$ and is given as

$$
\begin{aligned}
& \frac{\mathrm{d} G}{\mathrm{~d} t}=I+\alpha(A, G)-\beta(B, G)-\gamma(R, G), \\
& \frac{\mathrm{d} A}{\mathrm{~d} t}=A\left(\phi(G) h_{1}(A, B)-D_{(A)}\right), \\
& \frac{\mathrm{d} B}{\mathrm{~d} t}=B\left(\psi(G) h_{2}(A, B)-D_{(B)}\right) .
\end{aligned}
$$

The terms $\alpha(A, G)$ and $\beta(B, G)$ describe the effects of glucagon and insulin on the blood glucose concentration, respectively. Uptake of glucose by muscles and other tissues is represented by $\gamma(R, G)$, where $R$ is an externally determined quantity describing the the activity of the organism. The dependence of the insulin secretion rate on the blood glucose concentration is modelled by $\phi(G)$ and is a decreasing function of $G$. The function $\psi(G)$ represents the dependence of the glucagon secretion rate on the blood glucose concentration and is an increasing function of $G$. The two functions $h_{i}(A, B)(i=1,2)$ represent the mutual and self inhibitions of the secretion rates on the insulin and glucagon levels. The model is best used for illustrative purposes, but not necessarily for simulations. This illustrative model was the first model proposed where basal concentrations were 
treated as derivable quantities as opposed to explicitly defined fundamental parameters which are just assumed to exist.

The model proposed in this document is a three-by-three dynamical system with the following features: (a) the plasma (or extra-cellular fluid) represents a single pool in which glucose, insulin and glucagon enter and leave at prescribed rates; (b) increasing insulin concentration increases the rate at which glucose leaves the plasma, i.e. insulin promotes glucose uptake; (c) increasing glucagon concentration increases the rate at which glucose enters the plasma, i.e. glucagon promotes hepatic glucose production; (d) insulin secretion increases with increasing glucose concentration; (e) glucagon secretion increases with decreasing glucose concentration, and (f) insulin and glucagon disappear from the plasma independently of their action on the blood glucose level. Each unit process, such as the insulin secretion rate, depends on many variables and results from complex chemical processes. In order to model glucose metabolism using the fewest possible variables, each unit process is modelled using a 'black box' in which the output is a prescribed function of the input. The black-box functions chosen by Saunders et al. (1998) illustrate that a counter-regulatory glucose-insulin-glucagon system returns blood glucose to constant levels even after introducing large deviations (in either direction) of the blood glucose level. The authors demonstrate that under certain assumptions involving mutual and self inhibitions of the secretion of the counter-regulatory hormones, the glucose set point of the model is independent of the glucose input or glucose usage and the effectiveness of the hormones. The glucose set point is established by the ratio of rates of production and the rates of removal of the hormones from the blood. The authors use the language of engineering control to assign the name integral rein control to their proposed mechanism. To describe the control mechanism in the illustrative model of Saunders et al. (1998) and the model proposed in this study, we coin the term continuous rein control. Unlike the illustrative model of Saunders, Koeslag, and Wessels, the glucose set point of our proposed extensible model depends on both the insulin-dependent and insulin-independent glucose usage rates. We also do not require the model to demonstrate near zero steady-state error a priori.

\section{Model fundamentals}

Perhaps the most readily identifiable phenomena of the glucose regulatory system are the healthy body's ability to return the blood glucose concentration to a constant level after large inputs associated with eating and the body's ability to maintain the concentration despite large usage rates associated with exercise. The term basal state refers to the nearconstant levels of blood glucose, insulin, and glucagon that the body demonstrates for an extended period after absorption of a meal. Comparing parameter values derived by fitting nonlinear models to data gathered from individuals with different basal states might lead to misinterpretations of the processes occurring in each individual unless the model can determine why that individual has that particular basal state.

Non-zero basal levels of insulin and glucagon in the presence of non-zero disappearance rates clearly indicate that the secretion of these hormones is a continuous process with hormone secretion determined by the absolute blood glucose concentration and not determined by the difference of the concentration from basal values. Dependencies of basal values on other parameters (for example, the insulin sensitivity and the glucose threshold 
for glucagon secretion) provide important clues regarding the health of the individual. A complete model must be capable of distinguishing conditions leading to raised fasting plasma-glucose levels.

\section{Model construction}

In reviewing the literature on mathematical models of glucose regulation, it becomes obvious that no mathematical model except Saunders et al. (1998) has proposed that the basal glucose and insulin levels are derivable from the model itself. If one wants to construct a model of long-term glucose regulation, this key phenomena needs to be resolved and understood. We propose a starting mathematical model with the following features:

Exactly two hormones control glucose metabolism: insulin and glucagon. These hormones are catalytic in reactions involving blood glucose. That is, glucose uptake into the cells of the body occurs at a faster rate in the presence of insulin, but the insulin is not consumed in this process. The release of glucose into the blood stream occurs at a faster rate in the presence of glucagon, but the glucagon is not consumed in this reaction. Both insulin and glucagon disappear through a natural decay process independent of how the hormone is used to regulate the blood glucose.

Specific mathematical forms for the unit processes and any parameter values must be chosen. Our choice of unit processes are more realistic than the ones chosen by Saunders et al. (1998). Once the unit processes are established, different parameter values represent different individuals or states of health in a single individual. By changing the functional dependence of the rates of insulin and glucagon secretion or the functional dependence of the rates of glucose uptake and production, one describes a more complex model of hormone secretion and glucose regulation. The model is also designed to evolve by extension - such as adding a variable to model liver glycogen stores or accounting for both the fast and slow release of insulin. At each stage, more complex long-term behaviour is explored.

We start with a model designed to mimic the basic glucose-insulin-glucagon counterregulatory system. The initial three-by-three dynamical system is

$$
\begin{aligned}
\frac{\mathrm{d} B}{\mathrm{~d} t}= & -\dot{M}(B)+\dot{H}_{P}(G, B, I)-\dot{H}_{U}(I, B, G)-\dot{P}_{U}(I, B) \\
& -\dot{E}(t)+\dot{F}(t)+\dot{B}_{e x}(t) \\
\frac{\mathrm{d} I}{\mathrm{~d} t}= & -\delta_{I} I+\dot{Q}_{I}(B, I, G)+\dot{I}_{e x}(t), \\
\frac{\mathrm{d} G}{\mathrm{~d} t}= & -\delta_{G} G+\dot{Q}_{G}(B, I, G)+\dot{G}_{e x}(t),
\end{aligned}
$$

where $B$ (the blood glucose concentration in $\mathrm{mg} / \mathrm{dl}$ ), $I$ (the insulin concentration in $\mu \mathrm{U} / \mathrm{ml}$ ), and $G$ (the glucagon concentration in $\mathrm{pg} / \mathrm{ml}$ ) are the unknowns of the model. A single pool of extracellular fluid (volume $V_{f}$ ) is assumed, and the concentrations are the total quantity divided by this volume - e.g. $B=b / V_{f}$. The exogenous input rates per volume, $\dot{B}_{e x}(t), \dot{I}_{e x}(t)$, and $\dot{G}_{e x}(t)$, are prescribed during an experiment. The rates per volume that glucose leaves or enters the blood owing to exercise or food, $\dot{E}(t)$ and $\dot{F}(t)$, are also prescribed functions in the model. $\dot{H}_{P}$ is the rate per volume that glucose enters the blood owing to hepatic glucose production, $\dot{H}_{U}$ is the rate per volume that glucose leaves 
the blood owing to hepatic glucose uptake, $\dot{P}_{U}$ is the rate per volume of insulin-dependent peripheral glucose uptake, and $\dot{M}=\dot{m} / V_{f}$ is the rate per volume of insulin-independent glucose uptake. The secretion rates per volume of insulin and glucagon, $\dot{Q}_{I}$ and $\dot{Q}_{G}$, are predominantly functions of the blood glucose concentration, but depend on the insulin concentration and glucagon concentration if self and mutual inhibitions of secretion exist. The ordering of the arguments of each function implies the major dependencies of each term; for example, $\dot{Q}_{G}(B, I, G)$ implies that the glucagon secretion rate is mostly determined by the blood glucose concentration with some dependency on the insulin concentration and even less dependency on the glucagon concentration. The relative importance of these dependencies is still open to debate.

During this early stage of model development, we choose to keep the terms modeling the uptake and production of glucose as simple as possible, and we concentrate on models of insulin and glucagon secretion. Furthermore, we keep the models of secretion simple by considering only their dependence on the blood glucose concentration. Future reports will extend the model by more accurately modelling the uptake and production terms. The initial model is illustrative only and is not appropriate for fitting of data. The ability of the model's solution to fit experimental data only proves the solution's generality but does not prove the validity of the interpretation.

\section{The basic model}

In this first basic model under consideration, the secretion of the two hormones, insulin and glucagon, is dependent only on the blood glucose concentration. The rate of insulin secretion depends only on the blood glucose concentration with increasing blood glucose concentration leading to an increased rate of secretion; however, because it is plausible that the pancreas has a fixed capacity, the model assumes the existence of a maximum rate of insulin secretion regardless of the blood glucose level. No attempt is made to account for the two-phase insulin release that occurs during the intravenous glucose-tolerance test. Although inappropriate for a model of a complex organism, a one-compartment model for insulin is assumed, and the rate of glucose utilization in the body is assumed to be directly proportional to both the blood glucose concentration and the insulin concentration in this compartment. Under these assumptions, insulin sensitivity is a constant. The build up of insulin in the system is prevented by assuming a half-life for insulin, i.e. the level of insulin decays at a rate proportional to the amount of insulin present. The incorporation of insulin clearance proportional to blood insulin levels appears in several models (Berzins et al., 1986; Cobelli \& Pacini, 1988; Topp et al., 2000).

Including the effects of the counter-regulatory hormone glucagon allows for rein control of the blood glucose level. In this simple model, secretion of glucagon depends only on the blood glucose concentration with decreasing blood glucose concentration leading to increased secretion of glucagon. Similar to the insulin model, a maximum rate of glucagon secretion is assumed to exist, and the build up of glucagon in the system is prevented by assuming that the level of glucagon decays at a rate proportional to the amount of glucagon present. Furthermore, the rate of hepatic glucose production is assumed to be directly proportional to the glucagon concentration. The processes of glycogenolysis and gluconeogenesis are combined in this simple term and will be addressed more completely in future extensions of this model. 
The model considered in this paper is a special case of system (1)-(3) in three variables: the blood glucose concentration $B$, the glucagon concentration $G$, and the insulin concentration $I$. The hepatic production rate is directly proportional to the concentration of glucagon in the blood. The hepatic glucose uptake and the insulin-dependent peripheral uptake are combined into a single term such that the uptake rate is directly proportional to the product of the insulin concentration and the blood glucose concentration. The insulinindependent uptake is assumed to proceed at a constant rate. The central nervous system accounts for the majority of this insulin-independent uptake. The release of glucagon and the release of insulin into the blood are dependent on the concentration of glucose in the blood, and maximum release rates of these hormones are assumed to exist. The maximum release rate of glucagon occurs at zero blood glucose concentration, and the maximum release rate of insulin occurs for very large blood glucose concentration. The model is

$$
\begin{aligned}
& \frac{\mathrm{d} B}{\mathrm{~d} t}=\dot{F}(t)-\dot{E}(t)-\dot{M}_{0}+\alpha G-\beta B I, \\
& \frac{\mathrm{d} I}{\mathrm{~d} t}=-\delta_{I} I+Q_{I}^{\max }\left\{\frac{1}{2}+\frac{1}{2} \tanh \left(\frac{B-B_{I}}{r_{I}}\right)\right\}, \\
& \frac{\mathrm{d} G}{\mathrm{~d} t}=-\delta_{G} G+Q_{G}^{\max }\left\{\frac{1}{2}+\frac{1}{2} \tanh \left(\frac{B_{G}-B}{r_{G}}\right)\right\} .
\end{aligned}
$$

Initially, diet and exercise are included as first-order effects. Food is assumed to be a direct source of blood glucose and the rate food contributes to the glucose supply is modelled by $\dot{F}(t)$. In contrast, exercise is assumed to be a direct utilization of blood glucose and the rate exercise diminishes the glucose supply is modelled by $\dot{E}(t)$. Eleven parameters relate to the internal workings of an individual. $\dot{M}_{0}$ is the (constant) insulin-independent rate per volume of glucose uptake. The parameters $\delta_{I}$ and $\delta_{G}$ are related to the half-lives of the hormones, and account for the disappearance of insulin and glucagon respectively from the blood. The constant insulin sensitivity defined as $\frac{\partial}{\partial I}\left(\frac{\mathrm{d} B}{\mathrm{~d} t}\right) / B$ is modelled by $\beta$, and $\alpha$ represents the glucagon's effectiveness at releasing the glycogen stores and converting proteins to glucose. The development of insulin resistance is reflected in a lowering of the value of $\beta$, while the size of the glycogen stores and availability of substrates for gluconeogenesis affect the value of $\alpha$. The constants $Q_{I}^{\max }, B_{I}$, and $r_{I}$ relate to the secretion of insulin, and their values also reflect the health of the individual. Damage to the pancreatic cells that secrete insulin lowers the value of $Q_{I}^{\max }$ (the maximum rate of insulin release) or increases the values of $B_{I}$ and $r_{I}$, i.e. changes the pancreatic response to the blood glucose concentration. The constants $Q_{G}^{\max }, B_{G}$, and $r_{G}$ relate to the secretion of glucagon. Although not obviously as affected by the health of the individual, variations in this latter set of parameters might be responsible for the pre-diabetes condition of hyperinsulinemia in response to a slightly elevated basal glucose level. The parameters $B_{I}$ and $B_{G}$ are often referred to as 'threshold values' for insulin and glucagon secretion. With this terminology comes the assumption that the secretion of these hormones is insignificant until the blood glucose concentration reaches these threshold values; however, the basal level depends on the continuous balance between the non-zero secretion of the hormones during the basal state and the disappearance rates of the hormones. In the healthy individual, the blood glucose concentration only passes the threshold values during severe hypoglycemia or after significant glucose input such as provided by a carbohydrate meal. 


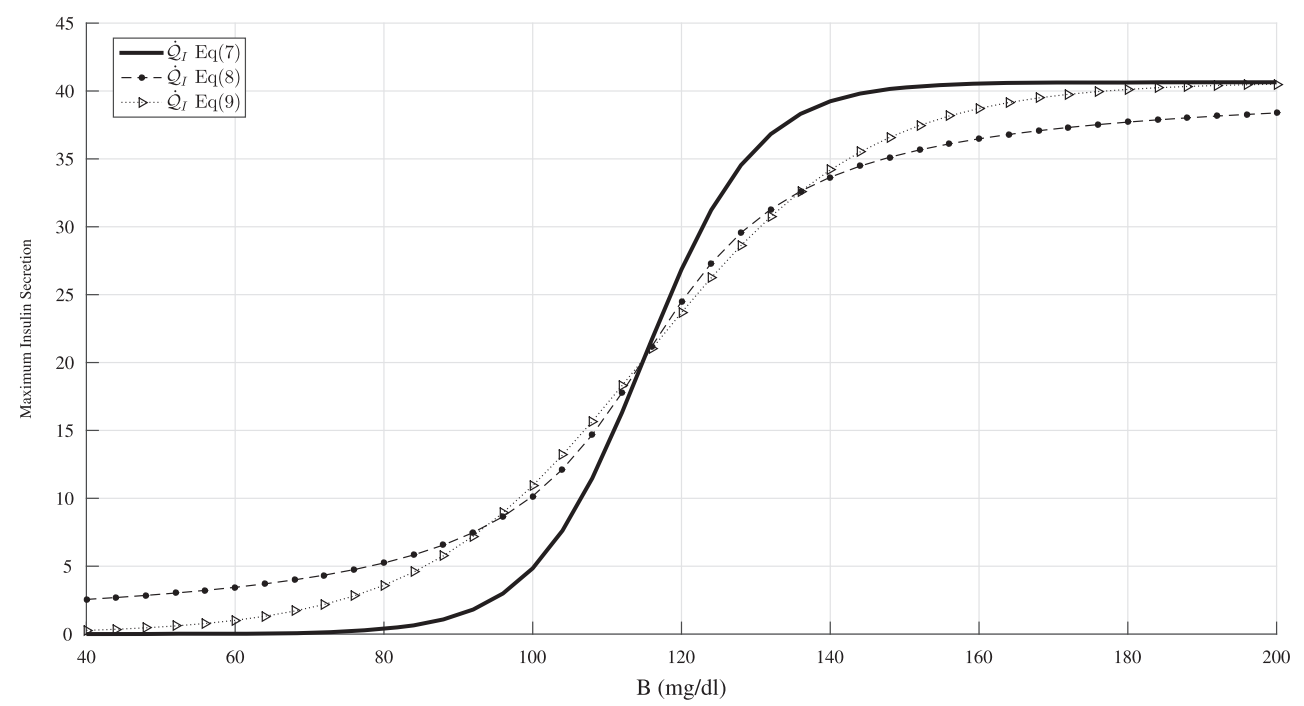

Figure 1. Three possible representations of insulin secretion, $\dot{Q}_{I}(B, I, G)$, represented by Equations (7)(9). Note: All curves generated using parameter values: $Q_{l_{i}}^{\max } \approx 40.644, B_{I_{i}}=115$, and $r_{l_{i}}=15(i=1 \ldots 3)$.

Each unit process, such as the insulin secretion rate, results from complex chemical processes. In order to model glucose metabolism using the fewest possible variables, the unit processes are modelled using 'black boxes' in which the outputs are prescribed functions of the inputs. As an example, consider the choice for the secretion rate of insulin. Following experimental observations, the dependency of the secretion rate on the blood glucose concentration is modelled as a strictly increasing function. Logically, a maximum rate of secretion at very large concentrations should exist, and the maximum secretion rate should be many times the normal basal secretion rate. Insulin secretion rates represented by sigmoidal functions are present in works by Cobelli, Pacini, and Salvan (1980), Rudenski et al. (1991), and Topp et al. (2000). Choosing a hyperbolic tangent to model insulin secretion is a common and reasonable choice, but not without consequences. For example, consider the three following functions as models of insulin secretion:

$$
\begin{aligned}
& \dot{Q}_{I}(B, I, G)=Q_{I_{1}}^{\max }\left\{\frac{1}{2}+\frac{1}{2} \tanh \left(\frac{B-B_{I_{1}}}{r_{I_{1}}}\right)\right\}, \\
& \dot{Q}_{I}(B, I, G)=Q_{I_{2}}^{\max }\left\{\frac{1}{2}+\frac{1}{\pi} \tan ^{-1}\left(\frac{B-B_{I_{2}}}{r_{I_{2}}}\right)\right\}, \\
& \dot{Q}_{I}(B, I, G)=Q_{I_{3}}^{\max } \frac{1}{1+\exp \left(-\frac{B-B_{I_{3}}}{r_{I_{3}}}\right)},
\end{aligned}
$$

where in a healthy individual $B_{I}>B_{\text {basal }}$. Figure 1 illustrates three different models of insulin secretion, represented by Equations (7)-(9), as functions of the glucose concentration $B$.

If $r_{I}$ is half of the difference between $B_{I}$ and $B_{\text {basal }}$, the maximum secretion rate is approximately 55 times the basal secretion rate for functional form (7) but is less than nine times the basal secretion rate for functional forms (8) and (9). Since the insulin secretion 
response in diabetics is different than the response in healthy individuals, discovering the changes in metabolism as the parameters $Q_{I}^{\max }, B_{I}$, and $r_{I}$ change is necessary. Each of the three functional forms (7)-(9) produce different results, and these differences help determine which of the forms is most appropriate.

The other unit processes undergo similar scrutiny, but at this early stage of modelling, perfection in describing each process is not sought. For most unit processes, choosing reasonable functional forms and extensively investigating the consequences of parameter variations is sufficient to discover qualitative trends. After this extensive analysis of the basic model, new phenomena are added one at a time, and the resulting model is investigated anew.

Having created the basic model, we must choose the values of the parameters and then investigate the effects of the various choices. For some choices, the model's solution mimics the time history of the blood glucose concentration in a functioning individual, whereas for other choices, the model's solution might predict impossibly low blood glucose concentrations. We attempt to choose parameter values that keep the model's solution within the range of human levels. Ideally, once the model is fully formed, many parameter values would be determined by fitting experimental data. We start with the following (rounded) constant values and use these values throughout the remainder of this paper:

- $\delta_{I}=.14 \mathrm{~min}^{-1}$ sets the half-life of insulin as a little less than five minutes,

- $\delta_{G}=.08 \mathrm{~min}^{-1}$ sets the half-life of glucagon as a little more than eight minutes,

- $V_{f}=13 \mathrm{l}=130 \mathrm{dl}$ is approximately the volume of the extracellular fluid in a $70 \mathrm{~kg}$ individual,

- $\dot{M}_{0}=100 \mathrm{mg} \mathrm{min}^{-1}$ is approximately $6 \mathrm{~g} \mathrm{~h}^{-1}$.

The other parameters vary in the course of the analysis; however, the analysis requires that a set of parameters consistent with the metabolism of a healthy individual be chosen in order to establish a baseline case. The effects of changing the parameter values from this base state are then examined. An initial set of parameter values is chosen to mimic healthy conditions for humans; however, no claim is made that any of the choices are the 'correct' choices. Also, it is not to be inferred that the particular values delineated below are the only values leading to healthy conditions. In fact, one purpose of the analysis is to determine the range of parameter values leading to a healthy individual. The baseline case parameters chosen are as follows:

- $B^{*}=90 \mathrm{mg} \mathrm{dl}^{-1}$ is the basal blood glucose concentration.

- $I^{*}=8 \mu \mathrm{U} \mathrm{ml}^{-1}$ is the basal insulin concentration.

- $G^{*}=120 \mathrm{pg} \mathrm{ml}^{-1}$ is the basal glucagon concentration.

- $\alpha G^{*}-\beta_{L} B^{*} I^{*}=170 \mathrm{mg} \mathrm{min}^{-1} / V_{f}$ is approximately $10 \mathrm{~g} \mathrm{~h}^{-1} / V_{f}$ and is equal to the net hepatic production rate per volume during basal conditions.

- $\beta_{M} B^{*} I^{*}=70 \mathrm{mg} \mathrm{min}-1 / V_{f}$ is approximately $4 \mathrm{~g} \mathrm{~h}^{-1} / V_{f}$ and is the insulin-dependent rate per volume of consumption of glucose external to the liver during basal conditions.

- The average value of $\dot{F}(t)$ is $\dot{F}_{0}=210 \mathrm{mg} \mathrm{min}^{-1} / V_{f}$ corresponds to a total daily carbohydrate intake equal to the Recommended Daily Allowance of $300 \mathrm{~g}$.

The above assumes that the rate per volume of insulin-dependent glucose consumption consists of two components: the conversion rate per volume of glucose to hepatic glycogen, 
$\beta_{L} B I$, and the use rate per volume of glucose by the muscles (and other tissues) of the body, $\beta_{M} B I$. To keep the model simple, the liver insulin concentration is assumed to be the same as the insulin concentration in the rest of the body. Thus, both effects are modeled using a single term, $\beta B I$ where $\beta=\beta_{L}+\beta_{M}$. Unfortunately, the decomposition of the insulindependent glucose consumption into two components adds an additional parameter to the system. At this level of modelling, choosing an accurate relationship between $\beta_{L}$ and $\beta_{M}$ is not possible since the apportionment of glucose between the compartments changes continuously and is dependent upon the state of glycogen storage in the liver and muscle. For simplicity, the relationship $\beta_{M}=2 \beta_{L}$ is chosen to hold throughout this analysis. Many of the other parameter values in the model are chosen in the next section where the basal conditions are examined in detail.

\section{Basal conditions and other steady states}

To assess the health of an individual, the fasting blood glucose concentration is measured. In a typical scenario, an individual experiences a 10-12 h overnight fast with blood drawn in the morning. The measured values are often (perhaps erroneously) referred to as the basal state. Here, we define the basal state as a fasting and resting state with energy used solely for cellular activity, respiration, and circulation. The basal values are nearly constant in the short term and represent a balance between the hepatic glucose production and the combination of the insulin-dependent and the insulin-independent glucose utilization. The fasting blood glucose concentration does not measure the basal state if the metabolism of prior food input takes longer than the 10-12 h overnight fast, especially when combined with the additional hepatic glucose production that usually occurs early in the morning. In all cases where the fasting level does not measure the basal level, the measured fasting level exceeds the intrinsic basal level. Thus, this measured quantity appropriately indicates the health of the individual and is suitable for diagnostic purposes, but substituting the fasting level for the basal level within a mathematical model leads to erroneous results when applied to individuals with poor glucose control.

The basal values, designated with an asterisk, are found from Equations (1) to (3) by setting the derivatives, $\dot{F}(t)$ and $\dot{E}(t)$ equal to zero. The values of $G^{*}$ and $I^{*}$ in terms of $B^{*}$ are

$$
I^{*}=\left(Q_{I}^{\max } / \delta_{I}\right)\left\{\frac{1}{2}+\frac{1}{2} \tanh \left(\frac{B^{*}-B_{I}}{r_{I}}\right)\right\}
$$

and

$$
G^{*}=\left(Q_{G}^{\max } / \delta_{G}\right)\left\{\frac{1}{2}+\frac{1}{2} \tanh \left(\frac{B_{G}-B^{*}}{r_{G}}\right)\right\},
$$

and when inserted into the steady-state version of Equation (4), $0=-\dot{M}_{0}+\alpha G^{*}-\beta B^{*} I^{*}$, yields

$$
\begin{gathered}
\left(\alpha Q_{G}^{\max } / \delta_{G}\right)\left\{\frac{1}{2}+\frac{1}{2} \tanh \left(\frac{B_{G}-B^{*}}{r_{G}}\right)\right\}=\dot{M}_{0} \\
+\left(\beta B^{*} Q_{I}^{\max } / \delta_{I}\right)\left\{\frac{1}{2}+\frac{1}{2} \tanh \left(\frac{B^{*}-B_{I}}{r_{I}}\right)\right\} .
\end{gathered}
$$


The model predicts that if the initial blood glucose concentration, the insulin concentration, and the glucagon concentration are exactly the above constant values then this constant state continues forever. The ability of an organism to perpetually maintain a constant state in the absence of an external energy source exposes one of the model's approximations: the model considers the liver to be a non-depletable source of glucose. Therefore, this model is inappropriate for studying metabolism under conditions where the body's store of excess glucose is depleted, for example, during periods of prolonged fasting or during periods of excessive exercise such as running a marathon. Accounting for the storage (and depletion) of glycogen in the liver and muscle requires an extension of the current model in which a steady-state solution under fasting conditions does not exist.

Equation (12) depends on all eleven model parameters related to the internal workings of the body. However, we may consider the grouping $\alpha Q_{G}^{\max } / \delta_{G}$ as a single parameter and the grouping $\beta Q_{I}^{\max } / \delta_{I}$ as another parameter. Taking this approach reduces the number of free parameters that determine the basal level to seven. Upon normalizing Equation (12), the number of free parameters is reduced to six. The normalization is not unique, and making a certain choice affects the ease of interpretation of the solutions. Since a disease state related to the under-secretion or ineffectiveness of the glucagon hormone is rare, we assume that, for each individual, the quantity $\alpha Q_{G}^{\max } / \delta_{G}$ remains constant. Normalization is achieved by dividing each term of Equation (12) by $\alpha Q_{G}^{\max } / \delta_{G}$ to get

$$
\frac{1}{2}+\frac{1}{2} \tanh \left(\frac{B_{G}-B^{*}}{r_{G}}\right)=\dot{\mathcal{M}}_{0}+\dot{\mathcal{Q}}_{I} \frac{B^{*}}{B_{0}^{*}}\left\{\frac{1}{2}+\frac{1}{2} \tanh \left(\frac{B^{*}-B_{I}}{r_{I}}\right)\right\},
$$

where

$$
\dot{\mathcal{M}}_{0}=\dot{M}_{0} /\left(\alpha Q_{G}^{\max } / \delta_{G}\right) \quad \text { and } \quad \dot{\mathcal{Q}}_{I}=\left(\beta B_{0}^{*} Q_{I}^{\max } / \delta_{I}\right) /\left(\alpha Q_{G}^{\max } / \delta_{G}\right)
$$

are dimensionless parameters. The choice of $B_{0}^{*}=90$ as the basal value of a healthy individual completes the normalization. The scaled rate of hepatic glucose production (left-hand side of Equation (13)) as a function of $B$ is plotted in Figure 2 using the values $r_{G}=30$ and $B_{G}=65,80,95,110$, and 125 on the same graph as the sum of the scaled insulin-independent usage rate and the scaled rate of whole-body glucose usage (right-hand side of Equation (13)) using various values of $\dot{\mathcal{Q}}_{I}$. The basal blood glucose concentration, $B^{*}$, is determined as the value of $B$ at the intersection point of a glucose production curve (decreasing functions) and a glucose consumption curve (increasing functions). As illustrated in Figure 2, variations of the basal blood glucose concentration value exist as the values $B_{G}$ and $\dot{\mathcal{Q}}_{I}$ vary when the values of $B_{I}$ and $r_{I}$ are held constant.

For discussion, making the additional choice of $B_{G}=80$ (the solid decreasing curve in Figure 2 to the specifications of a baseline individual, the parameter $\dot{\mathcal{M}}_{0}$ has the approximate value $\dot{\mathcal{M}}_{0}=.1655$. The value of $\dot{\mathcal{M}}_{0}$ remains constant throughout the following analysis. Figure 3 is the zoomed view of the intersections points of select glucose production and glucose consumption curves near $B^{*}=90$. Each subgraph demonstrates the variation of possible values of the parameters $B_{G}$ and $\dot{\mathcal{Q}}_{I}$ which may lead to a basal glucose concentration of $B^{*}=90$.

Without further information, choosing values of $B_{G}, r_{G}, B_{I}$, and $r_{I}$ describing a single baseline healthy individual is impossible. The additional information is to be found by analysing the response of the individual to glucose input. Until this analysis is performed, we introduce four baseline individuals to aid the discussion: 


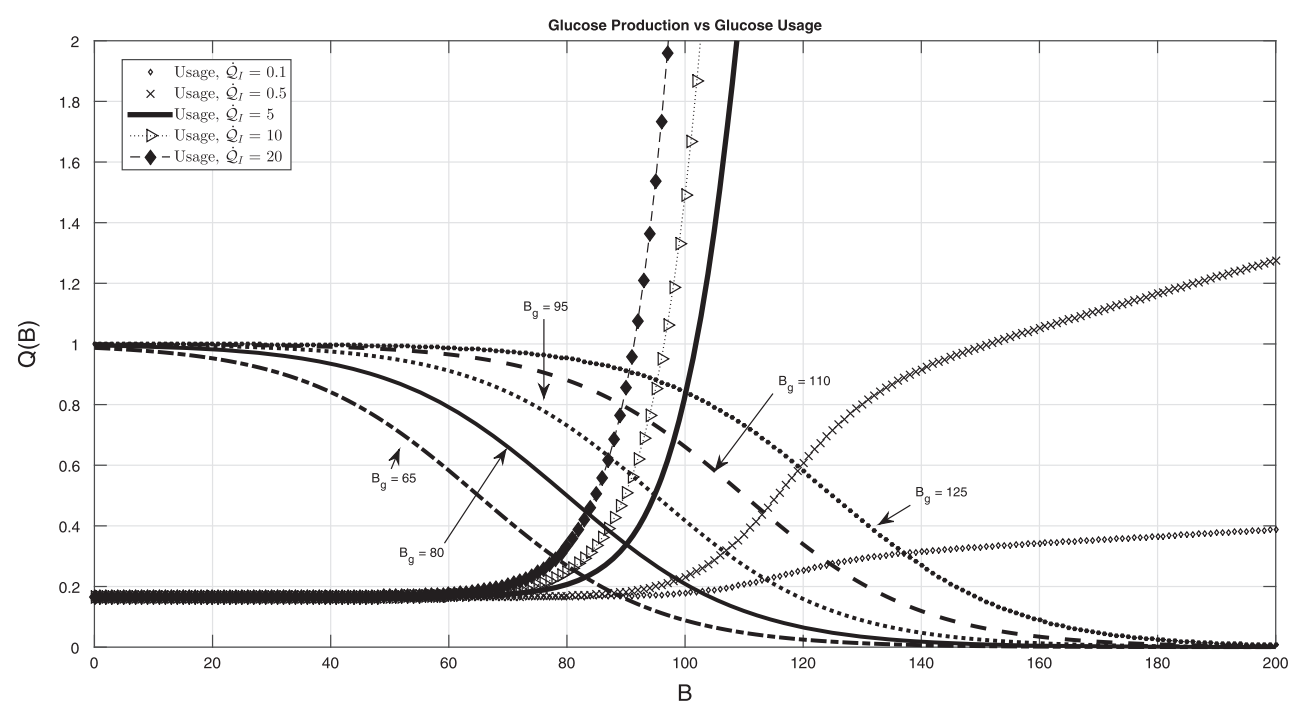

Figure 2. A closer examination of Equation (13).

Notes: Graphed is the basal glucose concentration defined by intersection of hepatic glucose production rate, the left-hand side of Equation (13) (illustrated by the decreasing curves $B_{G}=65,80,95,110,125$, from left to right respectively), and glucose-usage rate, the right-hand side of Equation (13) (illustrated by the increasing curves with $\dot{\mathcal{Q}}_{\mid}=.1, .5,5.0,10,20$, from left to right, respectively). Solid glucose-usage curve $\left(\dot{\mathcal{Q}}_{l}=5\right)$ intersects solid hepatic-production curve $\left(B_{G}=\right.$ $80, r_{G}=30$ ) at glucose concentration near $B^{*}=90$.
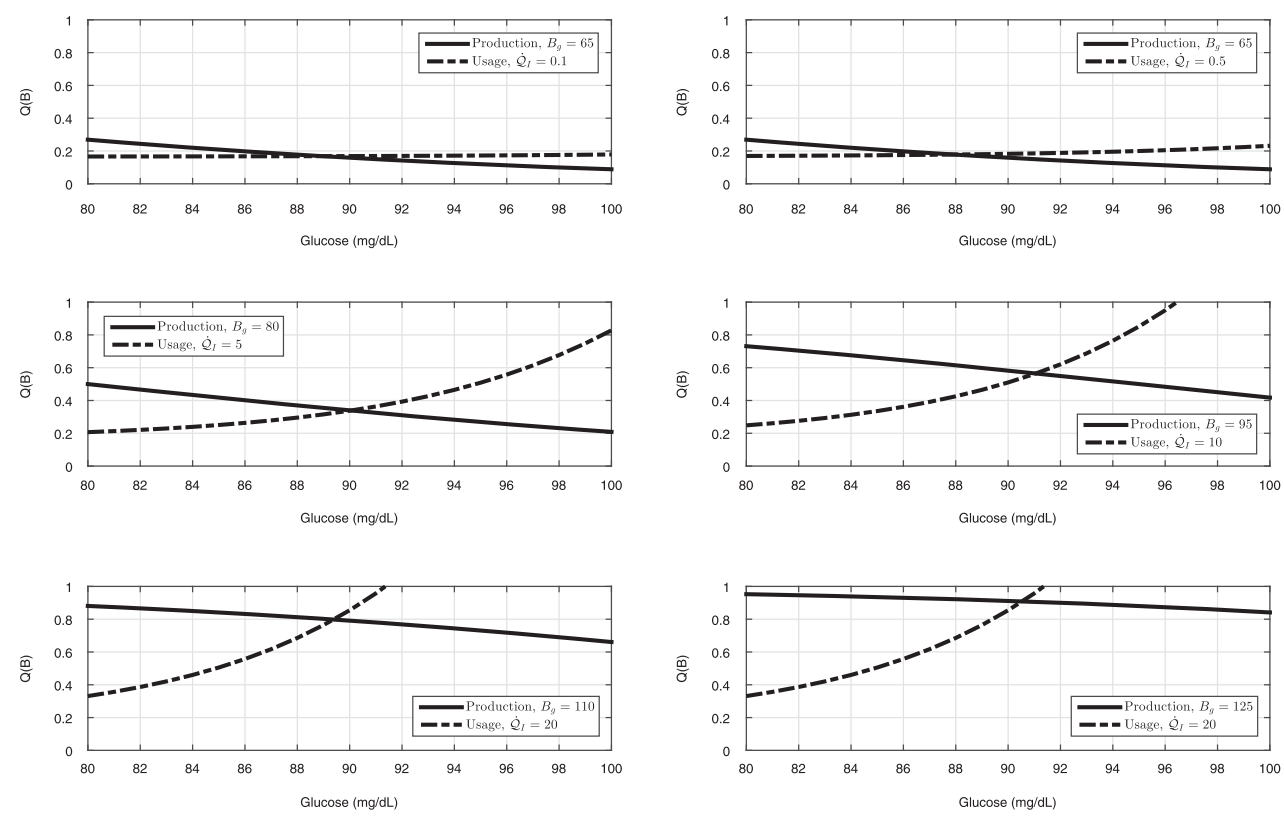

Figure 3. Zoomed-in views of Figure 2.

Notes: Hepatic glucose-production rate (decreasing curves) vs. glucose-usage rate (dash-dot curves) at glucose concentration near $B^{*}=90$.

- Baseline individual \#1: $r_{I}=15, B_{I}=105$, and $\dot{\mathcal{Q}}_{I}=1.5$;

- Baseline individual \#2: $r_{I}=15, B_{I}=115$, and $\dot{\mathcal{Q}}_{I}=5.0$; 

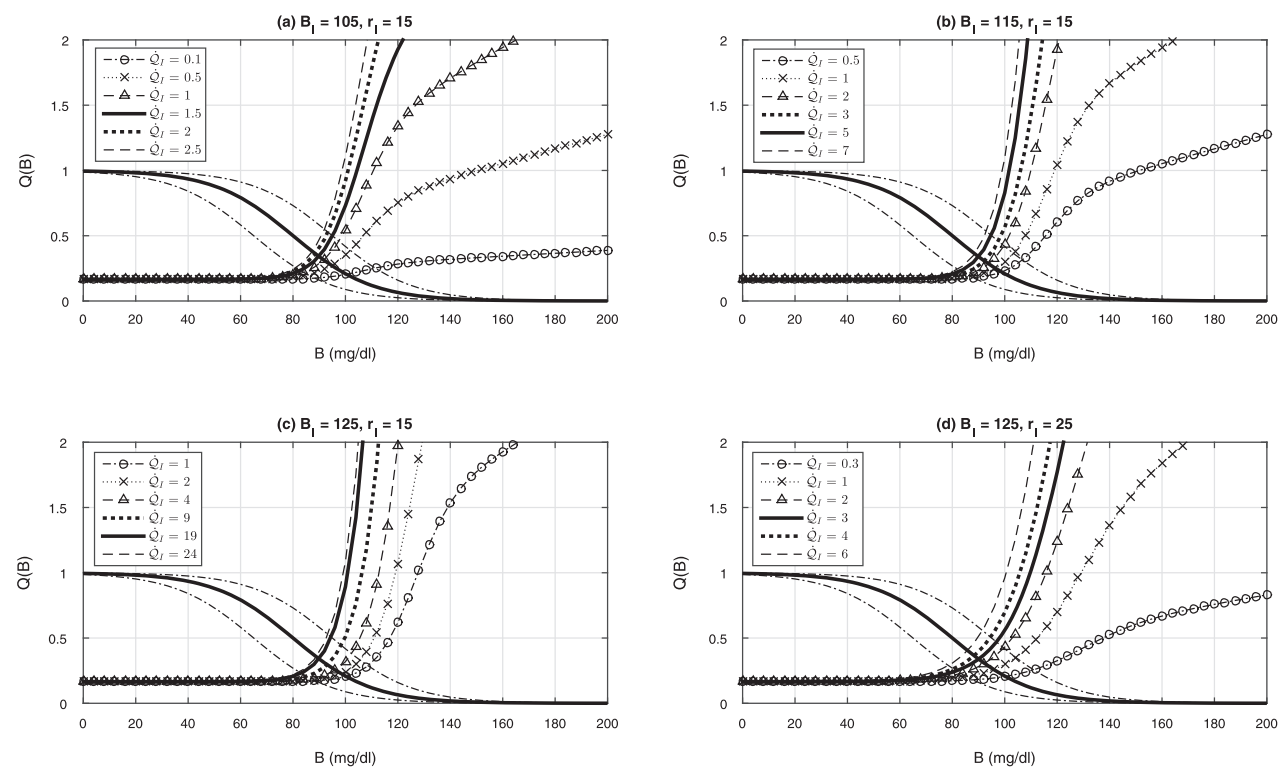

Figure 4. Basal glucose concentration defined by intersection of hepatic glucose-production rate (decreasing curves $\left.B_{G}=65,80,95\right)$ and glucose-usage rate (increasing curves). Parameter values: (a) $\dot{\mathcal{Q}}_{1}=.1, .5,1.0,1.5^{*}, 2.0,2.5 ;$ (b) $\dot{\mathcal{Q}}_{1}=.5,1.0,2.0,3.0,5.0^{*}, 7.0$; (c) $\dot{\mathcal{Q}}_{1}=$ 1.0, 2.0, 4.0, 9.0, 14.0, 19.0*, 24.0; and (d) $\dot{\mathcal{Q}}_{1}=.3,1.0,2.0,3.0^{*}, 4.0,6.0$. Solid glucose-usage curves (starred values of $\left.\dot{\mathcal{Q}}_{l}\right)$ intersect solid hepatic-production curve $\left(B_{G}=80, r_{G}=30\right)$ at glucose concentration near $B^{*}=90$.

- Baseline individual \#3: $r_{I}=15, B_{I}=125$, and $\dot{\mathcal{Q}}_{I}=19.0$;

- Baseline individual \#4: $r_{I}=25, B_{I}=125$, and $\dot{\mathcal{Q}}_{I}=3.0$;

where the (rounded) parameter values are chosen such that the glucose consumption curve intersects the baseline glucose production curve at approximately $B^{*}=90$ (see Figure 4).

Upon declaring a base state for each individual, the close-up view of Figure 2 provided in Figure 3 demonstrates the changes in basal conditions owing to three defects in the metabolism system: (a) insufficient suppression of glucagon secretion, (b) insulin resistance, and (c) insufficient insulin secretion.

The mechanism of glucagon secretion is typically one of suppression and not one of stimulation. For example, after large increases in the blood glucose concentration, the glucagon level drops to near zero as its secretion is almost completely suppressed. A defect in this suppression mechanism might be expressed as an increase in the value of $B_{G}$ causing secretion of glucagon to continue at larger values of the blood glucose concentration than normal. According to Figure 4, the basal blood glucose concentration increases moderately (about a $5-10 \%$ increase) as a result of this defect. The insulin response follows the hyperbolic tangent function, and the basal insulin concentration increases $50-100 \%$ over normal levels as a result of the slightly larger basal blood glucose concentration.

The second defect in the metabolism system demonstrated in Figures 2 and 3 is that of insulin resistance. In this model, insulin effectiveness is measured by the parameter $\beta$, and insulin resistance is expressed as a lowering of the $\beta$ value while holding all other parameters constant. In any individual, the ratio $\beta_{\text {eff }}=\beta / \beta_{\text {healthy }}$ is used to measure the degree of insulin resistance. For example, lowering the $\beta$ value by a factor of ten to 
twenty $\left(\beta_{\text {eff }}=.1\right.$ or .05$)$ represents the decrease in insulin effectiveness needed to increase the basal-glucose concentration from $B^{*}=90$ to $B^{*}=100$. Surprisingly, for constant $B_{G}=80$, the basal blood glucose concentration increases insignificantly, even as the effectiveness of the insulin approaches zero, $\beta_{\mathrm{eff}} \rightarrow 0$. Although the increase in basal blood glucose concentration to $B^{*}=100$ is relatively minor, the decrease in insulin effectiveness results in a significant increase in the basal insulin concentration - three to four times base-state levels. When the insulin-resistance defect combines with the glucagon-secretion defect basal blood glucose concentration only increases between 10 and $20 \%$ while the basal insulin concentration increases over six times the base-state concentration.

It is surprising that the basal level of blood glucose is only slightly increased by a significant decrease in the effectiveness of insulin. This contradiction is resolvable by considering that, in this model, the basal values are steady-state values which implies the situation has existed for a long time and continues indefinitely if the conditions remain the same. Thus, the basal values represent the body adjusting to the decrease in effectiveness of insulin by decreasing the glucagon concentration (i.e. lowering the hepatic production) and increasing the basal insulin level. In this model, even if the insulin effectiveness is zero, a steady state exists when the hepatic production rate matches the insulin-independent usage rate. Even though the basal glucose concentration is only mildly affected by a decrease in insulin effectiveness, the decrease in insulin effectiveness changes the transient response to an external glucose source.

The third defect, an insufficient secretion of insulin, is expressed in this model as a decrease in the maximum secretion rate $Q_{I}^{\max }$ and/or an increase in the threshold value $B_{I}$. Decreasing $Q_{I}^{\max }$ by a factor of ten has the same effect on the blood glucose concentration as decreasing the insulin effectiveness by a factor of ten; however, the basal insulin level declines as a result of the under-secretion of insulin. Likewise, increasing $B_{I}$ while holding all other parameters constant (see dotted curves in subfigures of Figure 3(a)-(c)) slightly increases the basal glucose concentration, decreases the basal insulin level and decreases the basal glucagon level.

The steady-state solutions corresponding to basal conditions are unsustainable since the body cannot constantly use energy (a non-zero value of $\dot{M}_{0}$ ) without an external energy source. Steady states under basal conditions exist for this model because the model contains the assumption that the liver is an infinite source of glucose. Although the assumption is obviously false for long-time solutions without additional energy inputs, the model reasonably predicts the variation of blood glucose concentration during short-term fasts. If a constant glucose input rate in excess of or equal to the resting energy usage rate is prescribed, then a sustainable steady-state solution in the resting individual exists. The term $\beta B I$ represents the removal of glucose from the blood with energy stored as fat in addition to energy stored as glycogen in the liver and muscle. The model does not account for the saturation of the storage sites, but the model should reasonably predict the variation of blood glucose concentration for time periods when saturation does not occur. Two special steady states are investigated below.

A set point exists for each individual where the input rate exactly matches the resting usage rate,

$$
F_{0}-\dot{M}_{0}-\beta_{M} B^{\#} I^{\#}=0
$$


(a) Energy Balance Glucose Production vs Glucose Usage

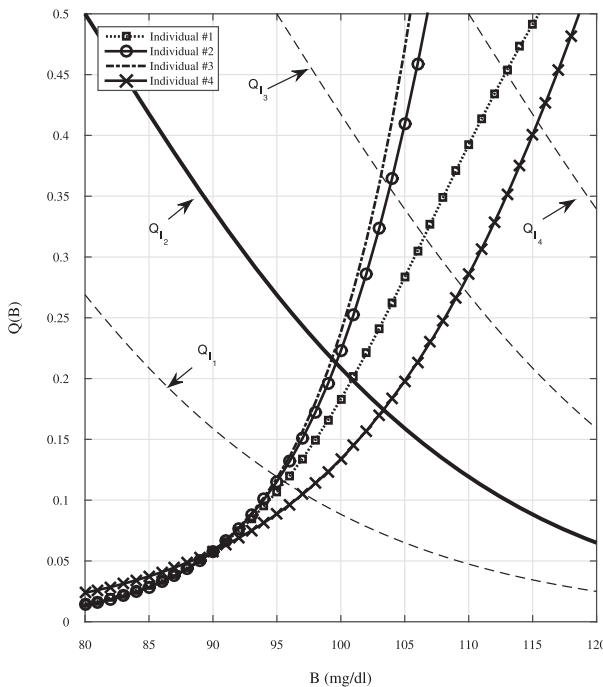

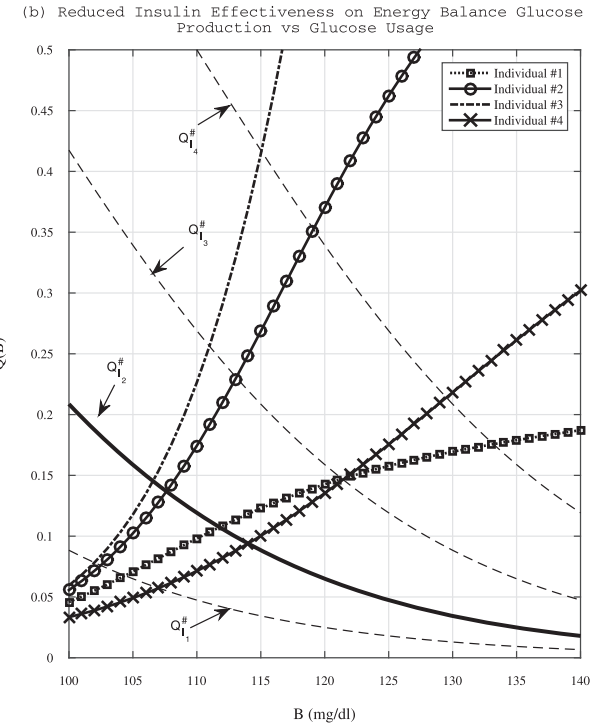

Figure 5. Energy-balanced set point: (a) healthy individuals, (b) insulin-resistant individuals ( $\beta_{\mathrm{eff}}=.25$ ).

and under these conditions, the net hepatic output is zero, i.e. $\alpha G^{\#}-\beta_{L} B^{\#} I^{\#}=0$. The scaled version of this last statement is

$$
\left\{\frac{1}{2}+\frac{1}{2} \tanh \left(\frac{B_{G}-B^{\#}}{r_{G}}\right)\right\}=\dot{\mathcal{Q}}_{I}^{\#} \frac{B^{\#}}{B_{0}^{*}}\left\{\frac{1}{2}+\frac{1}{2} \tanh \left(\frac{B^{\#}-B_{I}}{r_{I}}\right)\right\},
$$

where $\dot{\mathcal{Q}}_{I}^{\#}=\left(\beta_{L} Q_{I}^{\max } B_{0}^{*} / \delta_{I}\right) /\left(\alpha Q_{G}^{\max } / \delta_{G}\right)$. Both sides of the equation are plotted in Figure 5(a) for the four baseline individuals.

In baseline individuals \#1-\#3, the energy-balanced set point is given by $B^{\#} \approx 100$ and in baseline individual $\# 4, B^{\#} \approx 105$. Even with a defect in the glucagon suppression (larger values of $B_{G}$ ) the energy-balanced set point only modestly increases for these individuals. Much is learned about the parameter space by examining the result of reducing the insulin effectiveness by a factor of four $\left(\beta_{\text {eff }}=.25\right)$ in the baseline individuals (see Figure 5(b)). Baseline individuals \#2 and \#3 (larger values of $B_{I}$ along with a smaller value of $r_{I}$ ) demonstrate control of the value of $B^{\#}$ even when significant insulin resistance is combined with a glucagon-suppression defect. Baseline individuals \#1 and \#4 show a large increase in $B^{\#}$ with significant insulin resistance which is exacerbated by a glucagonsecretion defect.

The second special sustainable steady-state solution is the excess-energy set point, $B^{\circledR}$, which we define as the blood glucose concentration corresponding to the constant input rate $\dot{F}^{@}$ leading to an insulin level 99\% of maximum steady-state insulin level, $I^{@}=$ $.99 I_{\max }=.99 Q^{\max }{ }_{I} / \delta_{I}$. For this model, the excess-energy set point is $B^{@} \approx B_{I}+2.3 r_{I}$ leading to $G^{@} \approx 0$ and

$$
\dot{F}^{@}-\dot{M}_{0}-\beta B^{@} I_{\max } \approx 0 \text { or } \quad \dot{\mathcal{F}}^{@}-\dot{\mathcal{M}}_{0}-\dot{\mathcal{Q}}_{I} \frac{B^{@}}{B_{0}^{*}} \approx 0,
$$




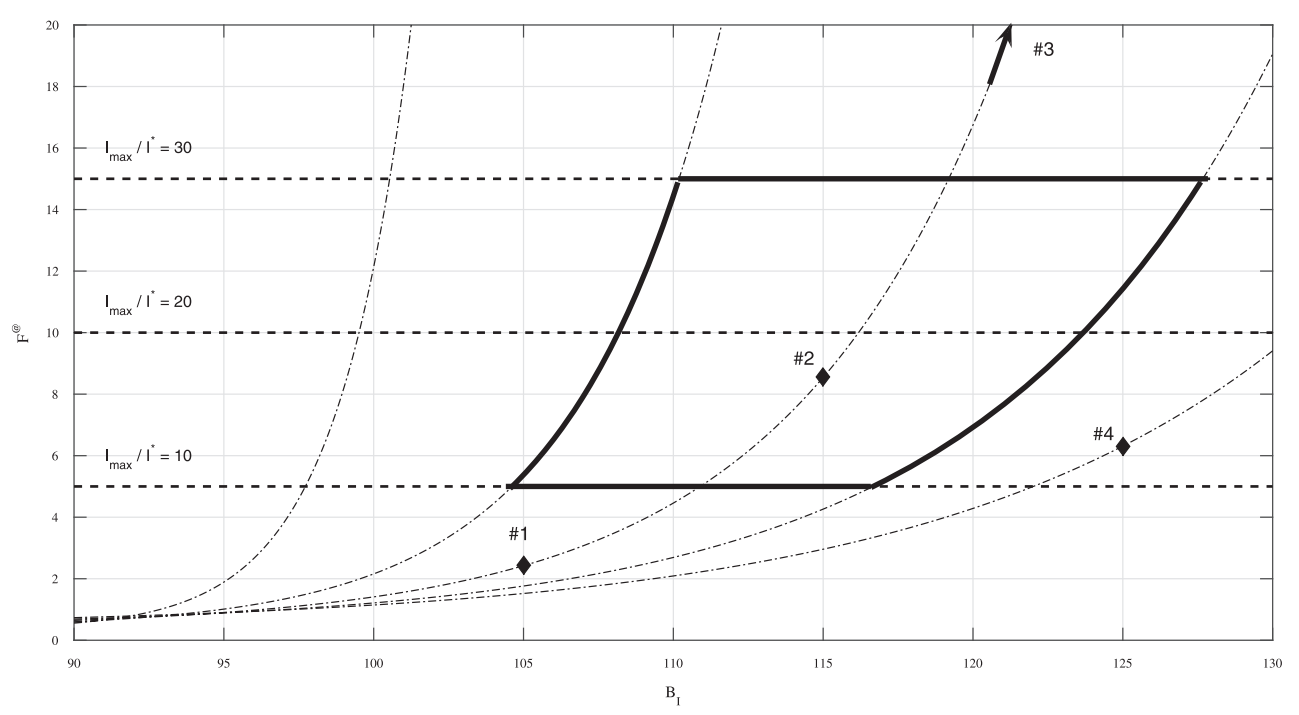

Figure 6. Excess energy input-rate for healthy individuals.

where scaling the former equation by $\alpha Q_{G}^{\max } / \delta_{G}$ produces the latter equation. The value of $\dot{\mathcal{F}}^{@}$ relates to basal conditions through Equation (13) and relates to the energy-balanced set point through Equation (14). The former relation is

$$
\dot{\mathcal{F}}^{@}=\dot{\mathcal{M}}_{0}+\frac{B^{@}}{B^{*}} \frac{\left\{\frac{1}{2}+\frac{1}{2} \tanh \left(\frac{B_{G}-B^{*}}{r_{G}}\right)\right\}-\dot{\mathcal{M}}_{0}}{\left\{\frac{1}{2}+\frac{1}{2} \tanh \left(\frac{B^{*}-B_{I}}{r_{I}}\right)\right\}} .
$$

Upon applying baseline conditions $\left(B^{*}=90, B_{G}=80\right.$, and $\left.r_{G}=30\right), \dot{\mathcal{F}}^{@}$ is a function of $B_{I}$ for constant $r_{I}$ (see Figure 6). Parameter values corresponding to the four baseline individuals are indicated on the graph. Baseline individuals \#2 and \#3 have larger values of $\dot{\mathcal{F}}^{@}$ and more control over their blood glucose level. Although theoretically possible to have infinite glucose control by prescribing a very large value to $B_{I}$, this situation is not found in nature. Choosing a parameter pair, $B_{I}$ and $r_{I}$, for fixed values of $B^{*}, B_{G}$, and $r_{G}$ is equivalent to choosing the ratio $I_{\max } / I^{*}$. Under the assumption $I_{\max }$ is achieved when $B \gg B^{*}$, it is reasonable to expect the ratio $I_{\max } / I^{*}$ to be large in healthy individuals. The region with $10<I_{\max } / I^{*}<40$ and $10<r_{I}<20$ is highlighted in Figure 6 and corresponds to our choice of approximate parameter values that would simulate a healthy individual. The restriction on $r_{I}$ also results from observations as to how the secretion rate depends on the blood glucose level.

Only baseline individual \#2 falls within the region that would simulate a healthy individual. Both individuals \#1 and \#4 are plausible; however, they both may exhibit difficulties in maintaining glucose control as their values of $I_{\max } / I^{*}$ are too low (based upon range determined in Figure 6). Individual \#3 also may additionally represent a functional individual, but the value of $I_{\max } / I^{*}$ is in excess of the 'healthy' range. Therefore, the parameter values associated with individual \#2 represent choices appropriate for a healthy individual and will be used as the baseline values for future analysis. 


\section{Conclusion}

The goal at the start of this investigation was to explore the usefulness of a simple model of the glucose-insulin-glucagon system as a base model for further exploration. For such a simple model to be useful, the solution must mimic the body's maintenance of near-constant concentrations during the postabsorptive period. This has clearly been demonstrated through the existence of a basal state which equates the hepatic glucoseproduction rate with the glucose usage rate. Although not presented, a stability analysis shows that all steady states discussed are indeed stable. In fact, the continuous rein control of the counter-regulatory naturally defines stable steady states. In this model, the basal state is determined as a derivable quantity based on the health of the individual without the restrictive assumptions of Saunders et al. (1998).

As emphasized, the results presented here are preliminary in nature, and every claim should be preceded with the words 'in this model'. The biggest flaw in the model, as in many other models, is the existence of a steady state solution which implies that the body maintains a constant blood glucose concentration even in the absence of external energy input. In reality, the body cannot survive without additional energy inputs. The contradiction is resolved if one realizes that the model predicts the liver to be an infinite source of energy which approximates the actual situation of the liver supplying energy at a near constant rate until an external energy source replenishes the store of glycogen in the liver. The next step in modelling and the topic of the next manuscript in this series will show that modelling the storage of glycogen in the liver allows for maintenance of nearly constant basal levels in the absence of a true steady state and that the model mimics the body's ability to process additional glucose in a timely manner while at the same time properly models the response to a long-term fast.

\section{Disclosure statement}

No potential conflict of interest was reported by the authors.

\section{ORCID}

Caleb L. Adams (D) http://orcid.org/0000-0002-8741-9239

\section{References}

Ackerman, E., Gatewood, L., Rosevear, J., \& Molnar, G. (1965). Model studies of blood glucose regulation. Bulletin of Mathematical Biophysics, 27, 21-24.

Ajmera, I., Swat, M., Laibe, C., Le Novère, N., \& Chelliah, V. (2013). The impact of mathematical modeling on the understanding of diabetes and related complications. CPT: Pharmaconmetrics \& Systems, Pharmacology, 2(54), 1-14.

Bergman, R., Ider, Y., Bowden, C., \& Cobelli, C. (1979). Quantitative estimation of insulin sensitivity. American Journal of Physiology - Endocrinology and Metabolism, 236(6), E667-E677.

Bergman, R., Phillips, L., \& Cobelli, C. (1981). Physiologic evaluation of factors controlling glucose tolerance in man: Measurement of insulin sensitivity and $\beta$-cell glucose sensitivity from the response to intravenous glucose. Journal of Clinical Investigation, 68, 1456-1467.

Bergman, R., Finegood, D., \& Ader, M. (1985). Assessment of Insulin Sensitivity in Vivo. Endicrine Reviews, 6(1), 45-86. 
Bergman, R. (2002). Pathogenesis and prediction of diabetes mellitus: Lessons from integrative physiology. The Mount Sinai Journal of Medicine, 69(5), 280-290.

Berzins, R., Tam, Y., Molnar, G., Rajotte, R., Wieczorek, K., McGregor, J., \& Fawcett, D. (1986). Pharmacokinetic approach to the estimation of hepatic removal of insulin. Pancreas, 1, 544-549.

Bolie, V. (1961). Coefficients of normal blood glucose regulation. Applied Physiology, 16, 783-788.

Borghouts, L., \& Keizer, H. (2000). Exercise and insulin sensitivity: A review. International Journal of Sports Medicine, 21(1), 1-12.

Brand-Miller, J. (2003). Gylcemic load and chronic Disease. Nutrition Reviews, 61(5), S49-S55.

Boutayeb, A., \& Chetouani, A. (2006). A critical review of mathematical models and data used in diabetology. BioMedical Engineering OnLine, 5(43). doi: 10.1186/1475-925X-5-43

Clark, D. (1997). Physical activity efficacy and effectiveness among older adults and minorities. Diabetes Care, 20(7), 1176-1182.

Cobelli, C., Pacini, G., \& Salvan, A. (1980). On a simple model of insulin secretion. Medical \& Biological Engineering \& Computing, 18, 457-463.

Cobelli, C., \& Pacini, G. (1988). Insulin secretion and hepatic extraction in humans by minimal modeling of C-peptide and insulin kinetics. Diabetes, 37, 223-231.

Della, C., Romano, M., Voehhelin, M., \& Seriam, E. (1970). On a mathematical model for the anallysis of the glucose tolerance curve. Diabetes, 19, 145-148.

Derouich, M., \& Boutayeb, A. (2002). The effect of physical exercise on the dynamics of glucose and insulin. Journal of Biomechanics, 35, 911-917.

Goldhaber-Fiebert, J., Goldhaber-Fiebert, S., Tristán, M., \& Nathan, D. (2003). Randomized controlled community-based nutrition and exercise intervention improves glycemia and cardiovascular risk factors in type 2 diabetic patients in rural Costa Rica. Diabetes Care, 26(1), 24-29.

Goodyear, L., \& Kahn, B. (1998). Exercise, glucose transport, and insulin sensitivity. Annual Review of Medicine, 49, 235-261.

Horton, E. (1988). Role and management of exercise in diabetes mellitus. Diabetes Care, 11(2), 201-211.

Leahy, J. (1990). Natural history of $\beta$-cell dysfunction in NIDDM. Diabetes Care, 42, 22-27.

Matthews, D., Hosker, J., Rudenski, A., Naylor, B., Treacher, D., \& Turner, R. (1985). Homeostasis model assessment: Insulin resistance and $\beta$-cell function from fasting plasma glucose and insulin concentrations in man. Diabetologia, 28, 412-419.

Roy, A., \& Parker, R. (2007). Dynamic modeling of exercise effects on plasma glucose and insulin levels. Journal of Diabetes Science and Technology, 1(3), 338-347.

Rudenski, A., Matthews, D., Levy, J., \& Turner, R. (1991). Understanding 'insulin resistance’: Both glucose resistance ain insulin resistance are required to model human diabetes. Metabolism, 40, 908-917.

Saunders, P., Koeslag, J., \& Wessels, J. (1998). Integral rein control in physiology. Journal of Theoretical Biology, 194(2), 163-173.

Serge, G., Turcogl, M., \& Varcellone, G. (1973). Modelling blood glucose and insulin kinetics in normal diabetic and obese subjects. Diabetes, 22, 94-97.

Sheard, N., Clark, N., Brand-Miller, J., Franz, M., Pi-Sunyer, F., Mayer-Davis, E., ... Geil, P. (2004). Dietary carbohydrate (Amount and Type) in the prevention and management of diabetes a statement by the American diabetes association. Diabetes Care, 27(9), 2266-2271.

Srinivasan, R., Kadish, A., \& Sridhar, R. (1970). A mathematical model for the control mechanism of free-fatty acid and glucose metabolism in normal humans. Computational Biometical Research, 3, 146-149.

Sturis, J., Polonsky, K., Mosekilde, E., \& Van Cauter, E. (1991). Computer model for mechanisms underlying ultradian oscillations of insulin and glucose. American Journal of Physiology Endocrinology and Metabolism, 260(5), E801-E809.

Švitra, D., Basov, I., \& Vilkyte, R. (2010). Modelling of glycaemia dynamics: Impact of physical exercises. Nonlinear Analysis: Modelling and Control, 15(2), 213-232. 
Sylvetsky, A., Edelstein, S., Delahanty, L., Walford, G., Boyko, E., Horton, E., ... Rother, K. (2016). Associations of dietary carbohydrates and carbohydrate subtypes with diabetes risk factors in the diabetes prevention program. Advances in Nutrition: An International Review Journal, 7(1), 14A.

Tirosh, A., Shai, I., Tekes-Manova, D., Israeli, E., Pereg, D., Shochat, T., ... Rudich, A. (2005). Normal fasting plasma glucose levels and type 2 diabetes in young men. New England Journal of Medicine, 353(14), 1454-1462.

Toffolo, G., Bergman, R., Finegood, D., Bowden, C., \& Cobelli, C. (1980). Quantitative estimation of of beta cell of beta cell sensitivity to glucose in the intact organism: A minimal model of insulin kinetics in the dog. Diabetes, 29, 979-990.

Topp, B., Promislow, K., DeVries, G., Miura, R., \& Finegood, D. (2000). A model of $\beta$-cell mass, insulin, and glucose kinetics: Pathways to diabetes. Journal of Theoretical Biology, 206, 605-619. 